\title{
COOL DUST IN THE OUTER RING OF NGC 1291
}

\author{
J. L. Hinz ${ }^{1}$, C. W. Engelbracht ${ }^{1}$, R. Skibba ${ }^{1}$, A. Crocker ${ }^{2}$, J. Donovan Meyer ${ }^{3}$, K. Sandstrom ${ }^{4}$, F. Walter $^{4}$, \\ E. Montiel ${ }^{1}$, B. D. Johnson ${ }^{5}$, L. Hunt ${ }^{6}$, G. Aniano ${ }^{7}$, L. Armus ${ }^{8}$, D. Calzetti ${ }^{2}$, D. A. Dale ${ }^{9}$, B. Draine ${ }^{7}$, \\ M. Galametz ${ }^{10}$, B. Groves ${ }^{4}$, R. C. Kennicutt ${ }^{10,1}$, S. E. Meidt ${ }^{4}$, E. J. Murphy ${ }^{7}$, and F. S. Tabatabaei ${ }^{4}$ \\ ${ }^{1}$ Steward Observatory, University of Arizona, 933 North Cherry Avenue, Tucson, AZ 85721, USA; jhinz@ as.arizona.edu \\ 2 Department of Astronomy, University of Massachusetts, Amherst, MA 01003, USA \\ ${ }^{3}$ Physics \& Astronomy Department, Stony Brook University, Stony Brook, NY 11794-3800, USA \\ ${ }^{4}$ Max-Planck-Institut fur Astronomie, Konigstuhl 17, D-69117 Heidelberg, Germany \\ 59 Institut d'Astrophysique de Paris, CNRS, Universite Pierre \& Marie Curie, UMR 7095, 98 bis bd Arago, F-75014 Paris, France \\ ${ }^{6}$ INAF-Osservatorio Astrofisico di Arcetri, Largo E. Fermi 5, I-50125 Fireze, Italy \\ ${ }^{7}$ Department of Astrophysical Sciences, Princeton University, Princeton, NJ 08544, USA \\ ${ }^{8}$ Spitzer Science Center, California Institute of Technology, Mc 314-6, Pasadena, CA 91125, USA \\ ${ }^{9}$ Department of Physics \& Astronomy, University of Wyoming, Laramie, WY 82071, USA \\ ${ }^{10}$ Institute of Astronomy, University of Cambridge, Madingley Road, Cambridge CB3 OHA, UK \\ Received 2012 April 18; accepted 2012 July 2; published 2012 August 17
}

\begin{abstract}
We examine Herschel Space Observatory images of one nearby prototypical outer ring galaxy, NGC 1291, and show that the ring becomes more prominent at wavelengths longer than $160 \mu \mathrm{m}$. The mass of cool dust in the ring dominates the total dust mass of the galaxy, accounting for at least $70 \%$ of it. The temperature of the emitting dust in the ring $(T=19.5 \pm 0.3 \mathrm{~K})$ is cooler than that of the inner galaxy $(T=25.7 \pm 0.7 \mathrm{~K})$. We discuss several explanations for the difference in dust temperature, including age and density differences in the stellar populations of the ring versus the bulge.
\end{abstract}

Key words: galaxies: individual (NGC 1291) - galaxies: photometry - galaxies: spiral

Online-only material: color figures

\section{INTRODUCTION}

Outer or external rings are typically large, low surface brightness features of barred and weakly barred galaxies, prominent at optical wavelengths. They are most frequently observed in early-type spirals (SO/a) and, as with gaseous rings and pseudorings, are most often believed to be associated with outer Lindblad resonances (Buta \& Combes 1996). These resonances are assumed to arise with bars or other perturbations (see simulations by, e.g., Bagley et al. 2009). For outer rings in galaxies without bars, explanations vary from ring creation through tidal forcing via interactions with companions or bars that have since dissipated to spiral density waves in the disk (Rautiainen \& Salo 2000). There are also outer rings that presumably formed in collisions (e.g., Appleton \& Struck-Marcell 1996) or through prograde major mergers of gas-rich disk galaxies (Brook et al. 2007).

Outer rings can have major axes that are twice the size of the bar component (Schwarz 1981) and, therefore, they dominate the outer areas of the disks of the galaxies that contain them. The study of outer disks as a path to understanding galaxy evolution has had recent renewed interest due to the discovery of very extended disks at ultraviolet (Gil de Paz et al. 2005; Thilker et al. 2005, 2007; Muñoz-Mateos et al. 2007), optical (de Jong et al. 2007; McConnachie et al. 2009), infrared (Engelbracht et al. 2004; Hinz et al. 2004, 2006), and submillimeter (Planck Collaboration et al. 2011) wavelengths. In particular, implications for the production source of dust and the mechanisms for heating and transporting the dust in galaxy outskirts may reveal much about the growth and chemical enrichment of disks.

The proximity of NGC 1291 and the wealth of available ancillary and space-based data make it an ideal test case for understanding dust emission in these extended disk structures. The first large outer ring structure to be discovered was, in fact, the one in NGC 1291 (Perrine 1922). The galaxy is classified as an (R)SB(s)0/a (de Vaucouleurs et al. 1991) with an inclination of $i=35^{\circ} \pm 7^{\circ}$ (Prescott et al. 2007) and is at a distance estimated to be between 7 and 10.4 Mpc (Masters 2005; Kennicutt et al. 2008); for the remainder of the paper we use the 10.4 Mpc value, following Kennicutt et al. (2011). In addition to its outer ring, at a radius of $\sim 9 \mathrm{kpc}$, at optical wavelengths it is characterized by a bright inner lens, a primary bar, and a small secondary bar misaligned by $\sim 30^{\circ}$ (de Vaucouleurs 1975; Pérez \& Freeman 2006; for more general information on the classification of rings and lenses in S0s, see, e.g., Michard \& Marchal 1993). Its star formation has been studied via $\mathrm{H} \alpha$ emission (Caldwell et al. 1991; Crocker et al. 1996; Meurer et al. 2006). It is part of the Spitzer Survey of Stellar Structure in Galaxies ( $\mathrm{S}^{4} \mathrm{G}$; Sheth et al. 2010) sample and, as such, has been re-classified in the Infrared Array Camera (IRAC; Fazio et al. 2004) bands as (R)SAB(l,ub)0 $0^{+}$(Buta et al. 2010). NGC 1291 is easily detected in the ultraviolet, but it is not classified as exhibiting an extended UV disk because optical emission is found to be coincident with the UV knots (Thilker et al. 2007). H I measurements of the galaxy (van Driel et al. 1988) show that the atomic gas is concentrated in the outer ring with a pronounced central hole. The H I gas mass is $0.81 \times 10^{9} M_{\odot}$, relatively gas-rich for an S0/a galaxy (e.g., Li et al. 2011).

Using images of the galaxy taken as part of the Spitzer Infrared Nearby Galaxies Survey (SINGS; Kennicutt et al. 2003) Legacy program, Bendo (2006) noted that the nucleus of NGC 1291 is the dominant source of $8 \mu \mathrm{m}$ emission, as well as 24 and $70 \mu \mathrm{m}$ warm dust emission, and that the 8 and $24 \mu \mathrm{m}$ emission are well correlated. However, at $160 \mu \mathrm{m}$, assumed to be associated with cool $(T \sim 20 \mathrm{~K})$ dust emission, the outer ring is a 
stronger source than the central portion of the galaxy. This was confirmed by Balloon-borne Large Aperture Submillimeter Telescope (BLAST) observations of NGC 1291, presented as part of a paper on resolved galaxies that also have Spitzer images (Wiebe et al. 2009). Both the central core and outer ring were detected by BLAST in a total of four observations of a $\sim 0.4 \mathrm{deg}^{2}$ area.

In a continuing effort to understand the role dust plays in the evolution of galaxies, we present Herschel Space Observatory images of NGC 1291, complementary to the Spitzer and BLAST images described above. Section 2 describes the observations and data reduction, and Section 3 the analysis and comparison with previous data. Section 4 contains a discussion of these results, while Section 5 contains a summary.

\section{OBSERVATIONS AND DATA REDUCTION}

The Multiband Imaging Photometer for Spitzer (MIPS; Rieke et al. 2004) images used here were observed as part of the SINGS Legacy project and reduced using version 3.06 of the Data Analysis Tool (DAT; Gordon et al. 2005). The DAT performs standard processing of infrared detector array data (e.g., dark subtraction, flat fielding) as well as steps specific to the MIPS arrays (droop correction). The images were calibrated using the most recent values available for MIPS data at all three wavelengths (Engelbracht et al. 2007; Gordon et al. 2007; Stansberry et al. 2007). The MIPS spatial resolutions are 6", $18^{\prime \prime}$, and $40^{\prime \prime}$ at 24,70 , and $160 \mu \mathrm{m}$, respectively.

Observations of NGC 1291 with the Herschel Space Observatory were taken as part of the Key Insights on Nearby Galaxies: a Far-Infrared Survey with Herschel (KINGFISH; Kennicutt et al. 2011) program and are described in detail in Engelbracht et al. (2010), Sandstrom et al. (2010), and Dale et al. (2012). Both Photodetector Array Camera and Spectrometer (PACS; Poglitsch et al. 2010) and Spectral and Photometric Imaging REceiver (SPIRE; Griffin et al. 2010) observations of NGC 1291 were acquired. PACS imaging was obtained in scan mode at the medium scan speed of $20^{\prime \prime} \mathrm{s}^{-1}$. The integrations achieved per pixel lead to approximate $1 \sigma$ surface brightness sensitivities of $\sigma_{\text {sky }} \sim 7,7$, and $3 \mathrm{MJy} \mathrm{sr}^{-1}$ at 70, 100, and $160 \mu \mathrm{m}$, respectively. SPIRE data were taken in Large-Map mode to 1.5 times the optical radius to depths of $3.2,2.5$, and $2.9 \mathrm{mJy} \mathrm{beam}^{-1}$, where the beam sizes are 423, 751, and $1587 \mathrm{arcsec}^{2}$, at 250, 350, and $500 \mu \mathrm{m}$, respectively. PACS and SPIRE images are the product of reduction with Herschel Interactive Processing Environment (HIPE) version 5.0.0 (Ott 2010). PACS data were also analyzed with Scanamorphos (Roussel et al. 2010; see Section 3). For PACS and SPIRE data, there is an additional modified background subtraction performed, in which the areas containing bright objects in the field are ignored when calculating the offset, but all other reductions are standard. This masked region was 7'.8 in radius for NGC 1291. NGC 1291 is located in an area of the sky with low infrared cirrus emission, so further image manipulation is not necessary. Figure 1 shows the final PACS, MIPS, and SPIRE images of NGC 1291.

Spitzer photometry used in this work is from the SINGS project compiled by Dale et al. (2007). PACS and SPIRE photometry is performed with a simple circular aperture, chosen to encompass the galaxy's mid-infrared light, after a median background subtraction determined from blank sky pixels away from the galaxy. All PACS and SPIRE photometric points are given a $15 \%$ uncertainty, including uncertainty due to the background subtraction, a $1 \%$ beam size uncertainty, and calibration uncertainties (5\% for PACS and 7\% for SPIRE as given by the Observer's Manuals of those instruments).

For the purposes of further analysis, we define the central or inner portion of the galaxy to be contained within a circle of radius $3^{\prime}(\sim 9 \mathrm{kpc})$. We define the ring to be in the aperture between circles of radii of $3^{\prime}$ and $6.5(\sim 20 \mathrm{kpc})$ from the center. Outside a radius of 6'.5 is considered to be off-galaxy (sky).

\section{RESULTS}

\subsection{General Image Description}

Comparing the morphology of NGC 1291 over a range of wavelengths allows us to determine the spatial distribution of the various stellar populations with respect to the spatial distribution of the dust (see Buta 2011 for a comprehensive review on the uses of morphology). Figure 1 shows that the typically identified features of the galaxy in the optical are also seen at infrared wavelengths. There is a bright inner-elongated, ovalshaped bulge, an area outside this bulge with very little flux, and an outer discontinuous ring marked with dense knots of emission of varying brightnesses, which are likely regions of recent star formation. The inner lens prominent at the $B$ band disappears in the infrared. In particular, the outer ring is faint relative to the bulge at bandpasses below $100 \mu \mathrm{m}$ and becomes more prominent at the $160 \mu \mathrm{m}$ and SPIRE wavelengths, consistent with findings in the proceeding by Bendo (2006). There are localized bright spots in the ring in the northwest and southeast arcs.

The resolution gains made by Herschel over Spitzer ( $5^{\prime \prime} .2$ for PACS versus $18^{\prime \prime}$ for MIPS at $70 \mu \mathrm{m}$ ) are illustrated by the level of detail in the central portion of the galaxy seen in the PACS image. Figure 2 shows the inner $3^{\prime} \times 3^{\prime}$ of both images. The PACS image has a bright central region surrounded by diffuse emission in the form of two extensions with a brighter patch on the northwest side. This is not visible in the $70 \mu \mathrm{m}$ MIPS image, where the lower resolution smears these details.

Given the larger beam size of the MIPS instrument and the difference in the operating temperatures of the two telescopes, it was anticipated that the $70 \mu \mathrm{m}$ and $160 \mu \mathrm{m}$ MIPS images would be more sensitive to diffuse emission, such as in the outer disks of galaxies, by a factor of five for dust emission at $T=15 \mathrm{~K}$ and by a factor of seven for dust emission at $T=20 \mathrm{~K}$ for a similar number of passes by each instrument (e.g., Hinz et al. 2008a). Figure 2 also shows an $11^{\prime} \times 11^{\prime}$ view, where the MIPS image has a faint detection of the ring at the northwestern and southeastern hot spots that PACS does not. This may be due to the intrinsic instrument differences as suggested above or due to the methods with which the PACS data are reduced currently. Highpass filtering used during the production of the PACS maps by HIPE may remove such diffuse features. Using the Scanamorphos software (Roussel et al. 2010; Roussel 2012) after an initial process with HIPE recovers some diffuse features for KINGFISH galaxies. This software produces maps after subtracting the thermal drift and the low-frequency noise of each bolometer purely by using the available redundancy. However, the outer ring of NGC 1291 does not appear at $70 \mu \mathrm{m}$ even when Scanamorphos is used. In this case, we find that is helpful to use both the PACS and MIPS complementary data sets for analysis. A discussion of apparent discrepancies between MIPS and PACS photometry can be found in Aniano et al. (2012).

\subsection{Star Formation in the Ring}

The contribution from the young stellar population to the emission from the ring can best be seen through a combination 

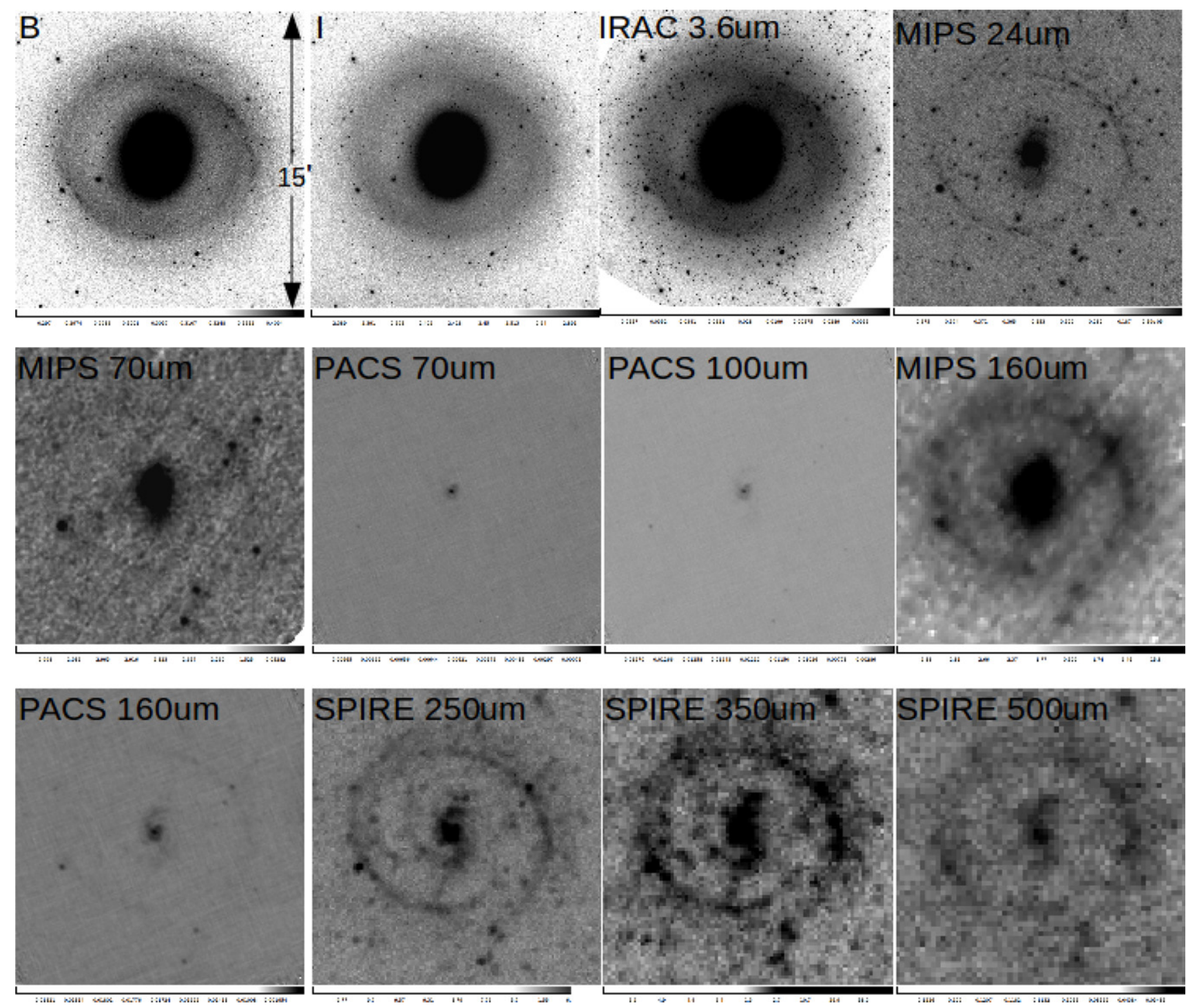

Figure 1. Images of NGC 1291. North is up and east is to the left. Starting in the top left corner the wavelengths are: $B, I$, IRAC $3.6 \mu \mathrm{m}$, MIPS $24 \mu \mathrm{m}$, MIPS $70 \mu \mathrm{m}$, PACS $70 \mu \mathrm{m}$, PACS $100 \mu \mathrm{m}$, MIPS $160 \mu \mathrm{m}$, PACS $160 \mu \mathrm{m}$, and SPIRE 250, 350, and $500 \mu \mathrm{m}$.

of ultraviolet imaging, with the near-UV (NUV) thought to trace the few hundred Myr stellar population, $\mathrm{H} \alpha$ imaging, tracing the $\leqslant 10 \mathrm{Myr}$ population, and infrared images at $24 \mu \mathrm{m}$, representing the warm dust emission generated near star-forming regions. Figure 3 shows GALEX NUV and far-UV (FUV) images (Gil de Paz et al. 2007), an $\mathrm{H} \alpha$ continuum-subtracted image (SINGS Legacy ancillary data), and the MIPS $24 \mu \mathrm{m}$ image (SINGS; Dale et al. 2007). NGC 1291 is not classified as an extended ultraviolet disk by Thilker et al. (2007) since the UV morphology is coincident with the optical morphology of the ring; this coincidence implies that there is a more evolved stellar component in the ring in addition to the young stars. The ring in $\mathrm{H} \alpha$ and $24 \mu \mathrm{m}$ is very thin $\left(\sim 40^{\prime \prime}\right.$ across at $\left.24 \mu \mathrm{m}\right)$ and is discontinuous, with discrete sources appearing mostly in arcs on the northwest and southeast sides of the galaxy. The UV images show many more star formation knots to the north and west of the main ring than the other wavelengths.

In a detailed study of $\mathrm{H}$ II regions in early-type-ringed galaxies by Crocker et al. (1996), NGC 1291 is considered to be the most unusual galaxy of their sample in $\mathrm{H} \alpha$ emission. They describe a diffuse and spiral-like $\mathrm{H} \alpha$ distribution in the inner region of the galaxy, which appears uncorrelated with the bar and lens features visible in optical images, with differing shape and orientation. The central emission also takes on this spiral-like appearance at $24 \mu \mathrm{m}$. Additionally, the $\mathrm{H} \alpha$ emission near the galaxy center is described by Crocker et al. (1996) as asymmetric, and weak $\mathrm{H} \alpha$ arms extend beyond the inner lens. These regions are filled with diffuse patterns of ionized gas filaments to a radius of $\sim 5 \mathrm{kpc}$, which is analogous to a pattern seen in the later type galaxy M 31 (Ciardullo et al. 1988) and M 81 (Devereux et al. 1995), and assumed to be caused by the ejection of the interstellar medium via a galactic wind. The diffuse and unusual $\mathrm{H} \alpha$ emission and the H I observations, showing an outer ring with a pronounced central hole (van Driel et al. 1988), have led to the supposition that there is little to no star formation in the central bulge (Hogg et al. 2001). However, we now see that the inner portion of the galaxy appears to be brighter compared to the ring at both $\mathrm{H} \alpha$ and $24 \mu \mathrm{m}$, making it unlikely that there is no star formation occurring centrally.

Global star formation rate estimates for NGC 1291 differ slightly with method. The rates are $\log \operatorname{SFR}\left(M_{\odot} \mathrm{yr}^{-1}\right)=$ $-0.5 \pm 0.4(\mathrm{H} \alpha ;$ Kennicutt et al. 2008), $-0.45 \pm 0.04$ 


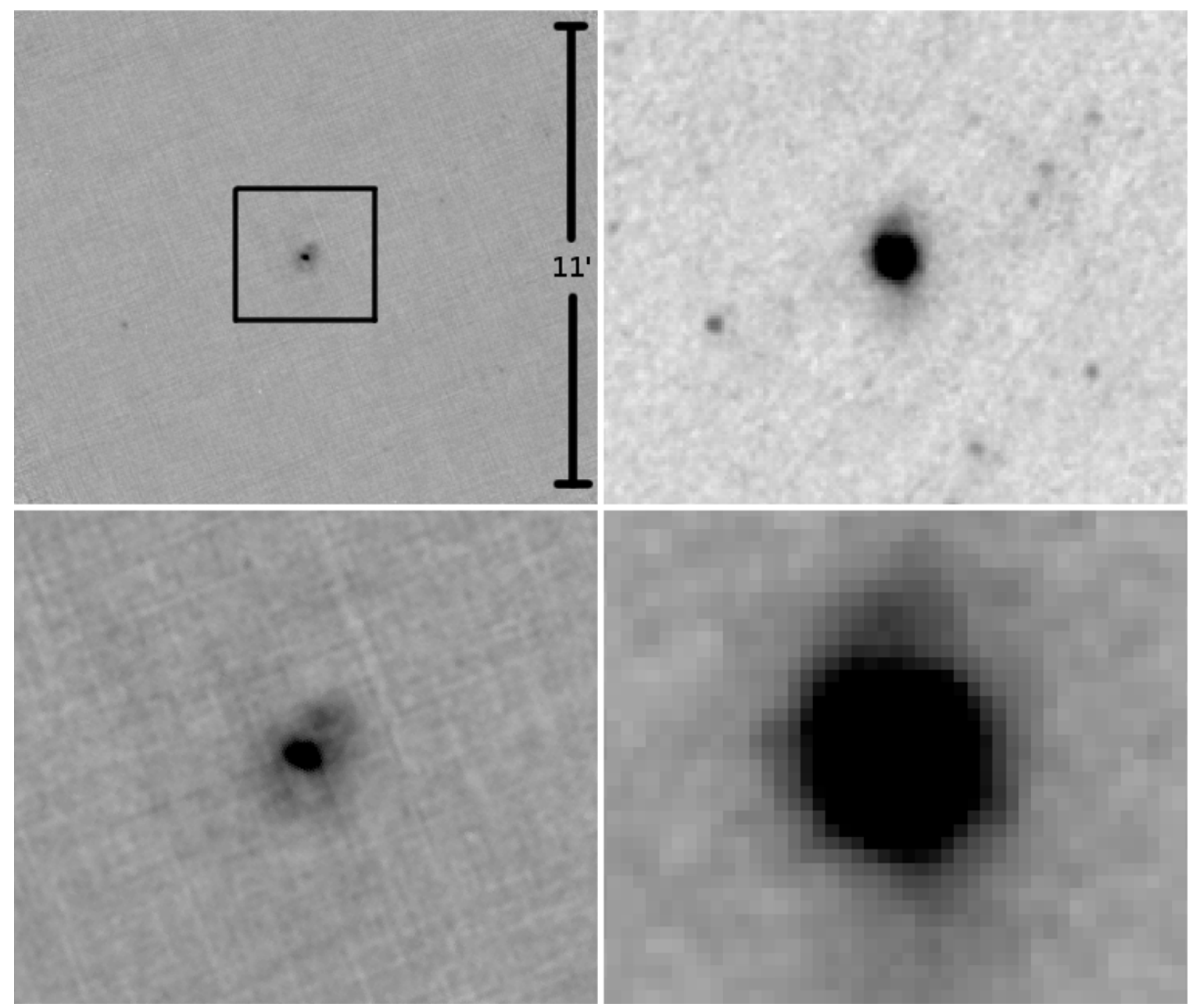

Figure 2. Top: PACS (left) and MIPS (right) $70 \mu \mathrm{m}$ images of NGC 1291. North is up and east is to the left, and the field of view is $11^{\prime}$. The MIPS image shows the outer ring while the PACS image does not. The box represents the field of view of the corresponding bottom image. Bottom: the same images zoomed in to a $3^{\prime}$ field of view, showing the resolution difference between the two instruments.

(FUV+TIR; Skibba et al. 2011), and $-0.07 \pm 0.05(\mathrm{H} \alpha+24 \mu \mathrm{m}$; Calzetti et al. 2010). While these star formation rates are within the known range for such galaxies (Kannappan et al. 2009; Skibba et al. 2011; D. A. Crocker et al., in preparation), the H I gas mass of NGC 1291 is higher than expected from its morphological type and is not coincident with any central star formation.

To understand the star formation rates specifically in the ring, we calculate rates using the GALEX UV data for this portion of the galaxy only. The FUV and NUV total magnitudes, within a circle of radius 6.5, are found to be 13.5 and 14.4, respectively, in agreement with the GALEX Atlas of Nearby Galaxies (Gil de Paz et al. 2007). For the aperture between $3^{\prime}$ and $6{ }^{\prime} .5$ that contains only the ring, the NUV magnitude is 15.1, which translates to a $\log$ star formation rate $\left(M_{\odot} \mathrm{yr}^{-1}\right)=-0.52$, following Donovan et al. (2009), who use a relation from Kennicutt (1998) for a similar early-type galaxy with an ultraviolet ring. Given the GALEX resolution and the distance to the galaxy and following Kennicutt (1989), we then calculate an average star formation rate density for the ring of $6.9 \times 10^{-5} M_{\odot} \mathrm{yr}^{-1} \mathrm{kpc}^{-2}$, with no corrections for reddening or dust extinction. Foreground extinction is estimated to be small here, only $A_{U}=0.07 \mathrm{mag}$ at $U$ band (Schlegel et al. 1998). Even if the extinction is two to three times larger than this estimate in the UV, the revised log star formation rate $\left(M_{\odot} \mathrm{yr}^{-1}\right)$ is only -1.44 , for a star formation rate density of $8.3 \times 10^{-5} M_{\odot} \mathrm{yr}^{-1} \mathrm{kpc}^{-2}$. This density is quite low for an outer ring (though similar to those derived for ESO 38147 and NGC 404; Donovan et al. 2009; Thilker et al. 2010) and may be due to the fact that the UV emission is discontinuous across the large area of the ring (i.e., between $3^{\prime}$ and 6.5).

\subsection{Cool Dust in the Ring}

Summing the sky-subtracted fluxes in the inner galaxy and in the ring area in each band and taking their ratio yields a simple measurement of the change in brightness of the central portion of the galaxy relative to the brightness of the ring. Figure 4 shows these ratios from 24 to $500 \mu \mathrm{m}$, where the errors are calculated from photometric uncertainties. We see that the ratio of the flux in the ring aperture to the flux in the central circle increases with increasing wavelength, indicating that there is a corresponding decrease in temperature of the dust in the ring compared to the inner galaxy.

Similarly, Figure 5 shows the spectral energy distribution (SED) of the whole galaxy at far-infrared wavelengths, along with the SED points for the inner portion of the galaxy only (within $3^{\prime}$ ) and for the ring only (between $3^{\prime}$ and 6.5). The SED for the ring shows that this area generates cooler emission than the central region of the galaxy. The central portion of the galaxy contributes more to the total galactic emission from warm dust at $24 \mu \mathrm{m}(\sim 60 \%)$ but is quickly overtaken by the cool ring starting at $160 \mu \mathrm{m}$, as already suggested 


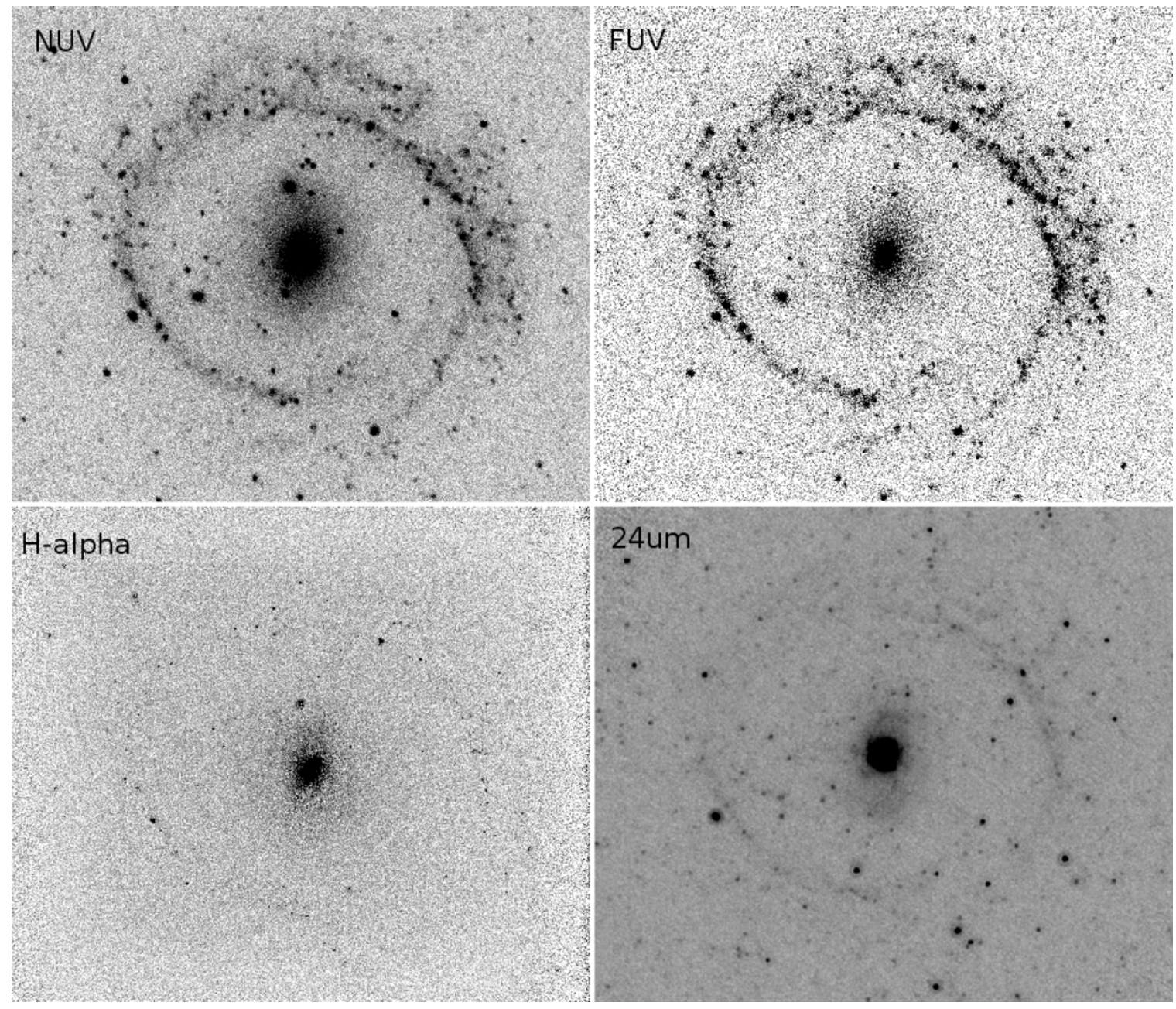

Figure 3. Images of NGC 1291 in the NUV and FUV, continuum-subtracted $\mathrm{H} \alpha$, and $24 \mu \mathrm{m}$. North is up and east is to the left.

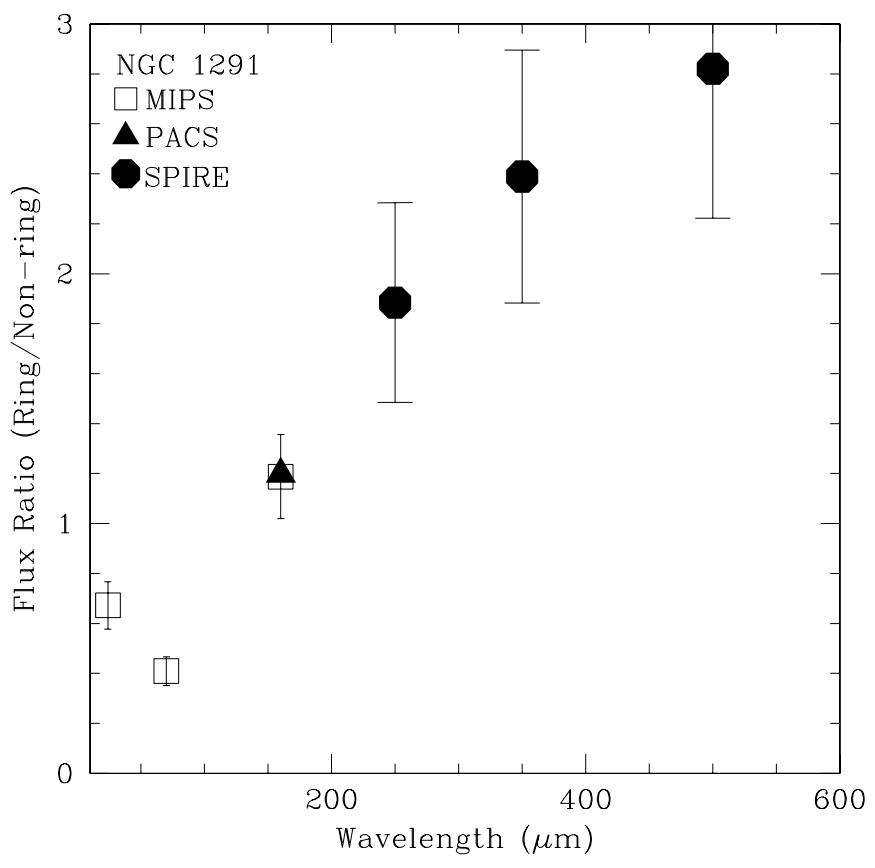

Figure 4. Ratio of the flux contained in the ring to the flux contained in the inner portion of the galaxy vs. wavelength. The central portion of the galaxy is that within a $3^{\prime}$ radius of the center. The ring is taken as the area between 3 and 6.5 from the center. Points are represented from PACS, MIPS, and SPIRE. Error bars are based on estimated photometric errors as stated in the text. by the results of Figure 4. To calculate the temperatures of the emitting dust in various portions of the galaxy, we fit a blackbody with a frequency-dependent emissivity with an exponent of 1.5 to both the ring and inner galaxy SEDs from 70 to $500 \mu \mathrm{m}$, following Engelbracht et al. (2010) and Skibba et al. (2011). Trends are unaffected if emissivity values of 1 or 2 are used, although the temperatures systematically increase or decrease, respectively. The uncertainties in the temperatures are computed via a Monte Carlo simulation in which 10,000 trials are performed. The photometric measurements are allowed to vary in a normal distribution with a standard deviation indicated by the photometric uncertainty. The cool dust temperature of the ring is $T_{\text {ring }}=19.5 \pm 0.3 \mathrm{~K}$ and for the inner portion of the galaxy it is $T_{\text {inner }}=25.7 \pm 0.7 \mathrm{~K}$. This gives $T_{\text {inner }} / T_{\text {ring }} \sim 1.3$.

Both of these dust temperature values are typical of cool dust temperatures in nearby galaxies (e.g., Hinz et al. 2006, 2008b). The values for NGC 1291 are slightly different from those found by Engelbracht et al. (2010), likely due to the difference in apertures used, but consistent with their overall finding that earlier type spirals have higher central temperatures by $20 \%$ to $50 \%$ over their late-type counterparts. The temperature ratio is closer to that found for strongly barred galaxies compared to those derived in weakly barred galaxies, though the bar classification for this galaxy changes slightly between optical and near-infrared analyses, from SB to SAB (Buta et al. 2010). 
Table 1

Dust Masses and Dust Mass Weighted Starlight Heating Intensities

\begin{tabular}{lccccr}
\hline \hline $\begin{array}{l}\text { Annular } \\
\text { Aperture } \\
(\text { arcmin })\end{array}$ & $M_{\text {dust }}$ & Area & $\langle U\rangle$ & $f_{\text {PDR }}$ & $q_{\text {PAH }}$ \\
\hline $0-1$ & $\left(M_{\odot} \mathrm{kpc}^{-2}\right)$ & $\left(\mathrm{kpc}^{2}\right)$ & $\left(U_{\odot}\right)$ & $(\%)$ & $(\%)$ \\
$1-2$ & $3.1 \mathrm{E}+04 \pm 1.7 \mathrm{E}+03$ & 28.8 & $4.6 \pm 0.3$ & $9.38 \pm 1.14$ & $1.60 \pm 0.20$ \\
$2-3$ & $2.1 \mathrm{E}+04 \pm 1.1 \mathrm{E}+04$ & 86.3 & $1.7 \pm 0.6$ & $7.72 \pm 2.05$ & $1.30 \pm 0.80$ \\
$3-4$ & $1.8 \mathrm{E}+04 \pm 7.9 \mathrm{E}+02$ & 143.8 & $0.7 \pm 0.2$ & $8.69 \pm 5.14$ & $1.30 \pm 1.20$ \\
$4-5$ & $3.1 \mathrm{E}+04 \pm 5.7 \mathrm{E}+03$ & 201.3 & $0.5 \pm 0.1$ & $7.76 \pm 1.13$ & $2.40 \pm 0.70$ \\
$5-6$ & $4.1 \mathrm{E}+04 \pm 1.4 \mathrm{E}+04$ & 258.8 & $0.4 \pm 0.1$ & $7.49 \pm 3.59$ & $3.00 \pm 1.30$ \\
\hline
\end{tabular}

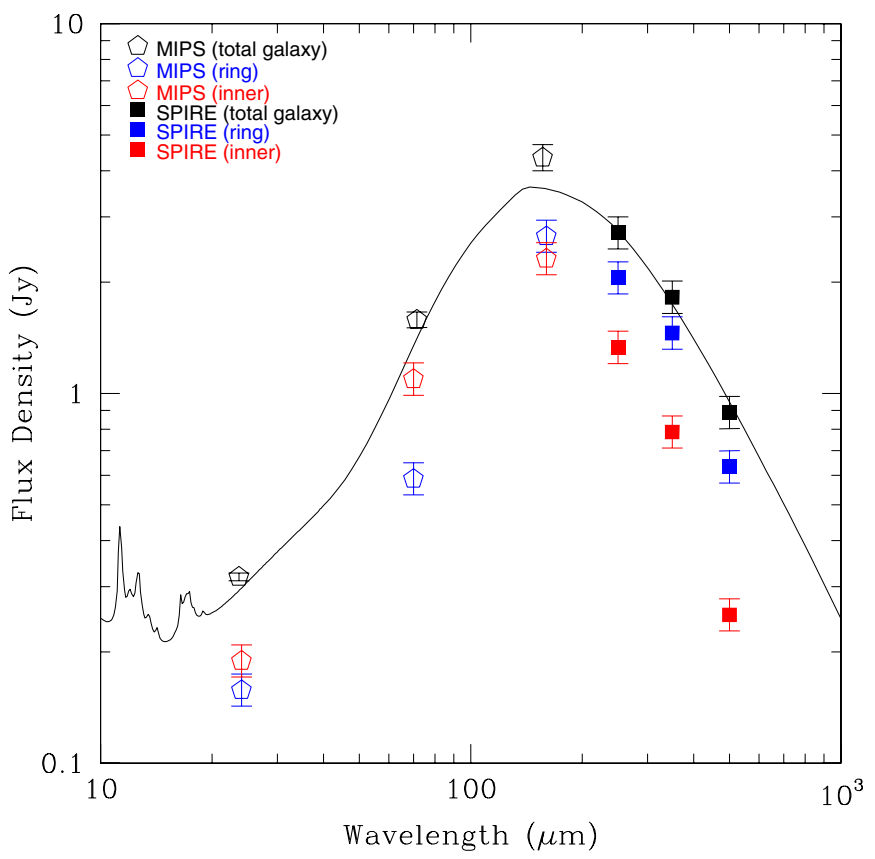

Figure 5. SEDs of the inner portion of NGC 1291, the outer ring of NGC 1291, and NGC 1291 in total. Open pentagons in all colors are MIPS data points. Solid squares in all colors are SPIRE data points. Black points represent the total SED of the galaxy. Red points represent the SED of the inner portion of the galaxy, while blue points represent the SED of the ring only. The black solid line is a model fit to the total SED (all black points) using results from Aniano et al. (2012).

(A color version of this figure is available in the online journal.)

The dust masses for NGC 1291, as a whole and for its components, can be calculated using the mid-infrared photometry from IRAC, PACS, MIPS, and SPIRE and the models of Draine $\& \operatorname{Li}(2001,2007)$ and constructed as suggested in Draine et al. (2007), using a mixture of amorphous silicate grains and carbonaceous grains with a distribution of grain sizes. All images are convolved to the MIPS $160 \mu \mathrm{m}$ resolution before modeling (Aniano et al. 2011). Details of the use of these models specifically with Herschel photometry are described extensively in Aniano et al. (2012) for two other spiral galaxies from KINGFISH. The results of such fitting for NGC 1291 are summarized here, with the black solid line in Figure 5 representing the final fit to the data for the whole galaxy. The apertures, dust masses $\left(M_{\text {dust }}\right)$, aperture areas, mean starlight heating intensities $(\langle U\rangle)$, fluxes radiated by dust corresponding to photodissociation regions $\left(f_{\mathrm{PDR}}\right)$, and the fractions of grain mass contributed by polycyclic aromatic hydrocarbons $\left(q_{\mathrm{PAH}}\right)$ are listed in Table 1. The total dust mass for the galaxy, calculated from a sum of the modeling for each individual galaxy pixel, is $M_{\text {dust }}=2.8 \times 10^{7} \pm 3.5 \times 10^{6} M_{\odot}$, with a total dust luminosity of $L_{\mathrm{dust}}=2.4 \times 10^{9} \pm 2.3 \times 10^{7} L_{\odot}$. Dust masses calculated for annular apertures with radii in intervals of $1^{\prime}$ out to $6^{\prime}$ show that the dust in the ring dominates the total dust mass of NGC 1291. The average starlight heating intensity is calculated to be the largest in the inner portion of the galaxy. This $\langle U\rangle$ can be related to the characteristic dust temperature, $T_{\text {dust }}$, by the approximate relation $T_{\text {dust }}=18 \mathrm{~K} \times\langle U\rangle^{1 / 6}$, where $\langle U\rangle$ is in units of the starlight energy density in the solar neighborhood (Aniano et al. 2012).

\section{DISCUSSION}

Several explanations for the existence of an outer ring with cooler dust may be plausible. It is possible that all spiral galaxies have a dust temperature gradient between their inner and outer disks. This might be due to the concentration of old stars or star formation occurring at the center of the galaxy in comparison to the more sparse stellar population of the comparatively large outer disk, i.e., that the density of the radiation field in the center of a galaxy is higher than that in the outer parts. Another explanation could be that the nucleus of the galaxy contains an active galactic nucleus (AGN) which heats the dust to higher temperatures in the inner region in comparison to the outskirts. Perhaps the inner bar of the galaxy fuels star formation in that area by funneling gas continuously, such that rapid star formation heats the inner dust to warm temperatures. Or it could be that the stellar population of the outer ring is older than that of the inner features. In this case, the older stars maintain the dust at cooler temperatures than areas with active star formation. We now discuss each of these possible explanations in turn.

\subsection{Temperature Gradients}

First, we explore the idea that all galaxies have similar dust temperature gradients to NGC 1291. Engelbracht et al. (2010) showed that the central areas of early-type spiral galaxies generally have enhanced dust heating of their cool dust components compared to their disks. They find that, on average, the cool dust temperature of the central component is $15 \% \pm 3 \%$ hotter than the disk. Similarly, Pohlen et al. (2010) showed that SPIRE surface brightness ratios seem to decrease with radius in spirals, implying that the dust in the outer regions is colder than dust in the centers of galaxies. Galametz et al. (2012) study 11 galaxies in the KINGFISH sample, including NGC 1291, fitting Spitzer and Herschel data SEDs with two modified blackbodies and creating spatially resolved maps of their dust properties. They also see systematic drops in dust temperature with radius for disk galaxies, also on the order of $10-15 \mathrm{~K}$ from inner to outer galaxy.

However, it is not obvious that NGC 1291 should follow this pattern and that outer rings should be dominated by cool 

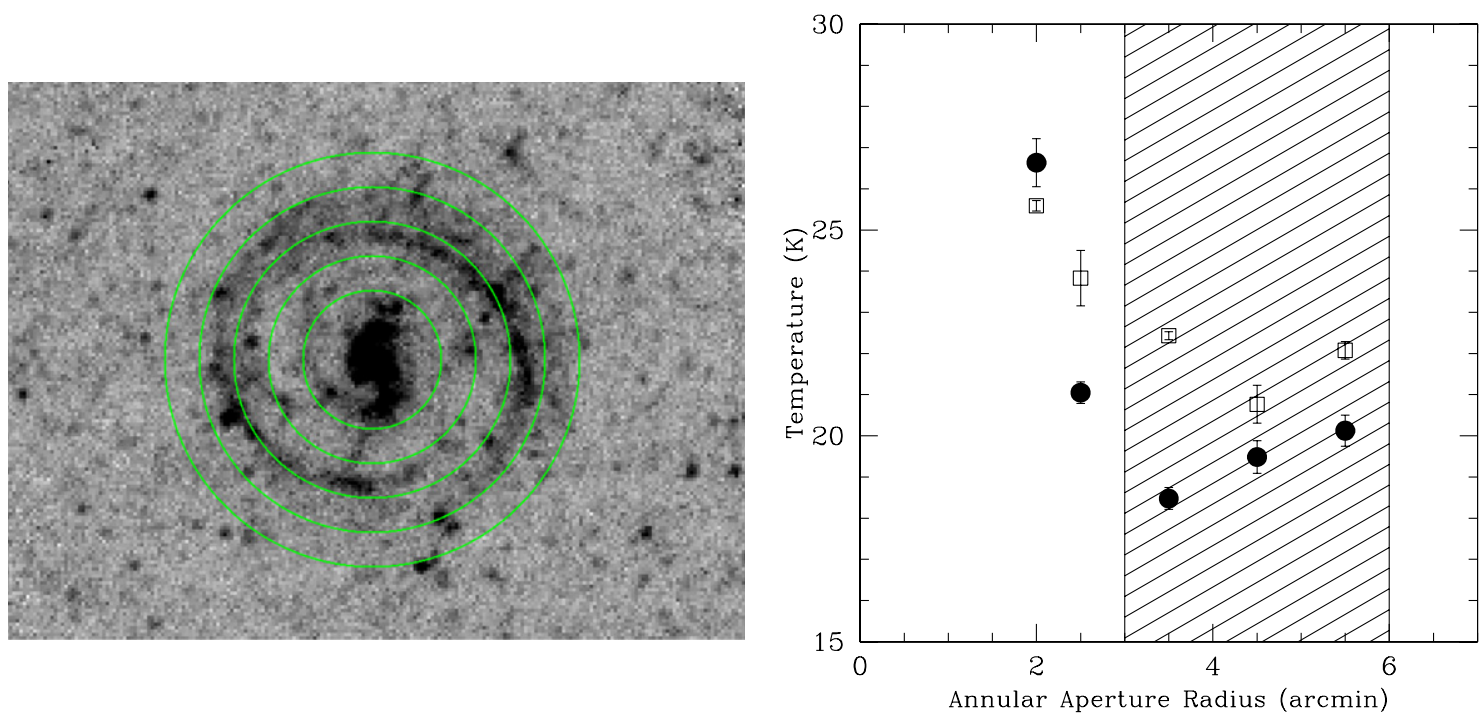

Figure 6. Left: the $250 \mu \mathrm{m}$ image of NGC 1291 (gray scale) overlaid with apertures at $2^{\prime}, 3^{\prime}, 4^{\prime}, 5^{\prime}$, and $6^{\prime}$ radii (green circles). Right: the dust temperature deduced from the infrared flux densities within each aperture. Solid dots represent NGC 1291 while open squares represent NGC 628, a comparison face-on spiral galaxy. The shaded region indicates the approximate area occupied by the ring of NGC 1291 at SPIRE wavelengths.

(A color version of this figure is available in the online journal.)

dust emission. UV rings in S0 and early-type spiral galaxies are often associated with sites of recent star formation, sometimes marked by clumpy H II regions, and can even be the only sites of star formation in some galaxies (e.g., Donovan et al. 2009; Thilker et al. 2010). A recent study of five nearby barred S0 galaxies with rings by Marino et al. (2011) showed that these outer rings account for a majority (up to 70\%) of the flux at UV wavelengths, indicating their young stellar population. Buta et al. (2010) specifically discuss the morphology of NGC 1291 based on the $B$ band and $3.6 \mu \mathrm{m}$ IRAC images (Sheth et al. 2010), saying that the outer ring is "where most of the recent star formation is taking place" and is prominent in the $B$ band (Figure 1). At $3.6 \mu \mathrm{m}$, however, the ring does not stand out and is instead a broad ellipse in a rounder diffuse background (Figure 1 and their Figure 7). Therefore, we might expect that, if the $24 \mu \mathrm{m}$ emission is associated with the warm $(T \sim 50 \mathrm{~K})$ dust heated by the young stellar population (seen at UV and $B$ band to be prominent), then the warm dust at $24 \mu \mathrm{m}$ should be brighter in the ring as well, yet the wavelengths associated with cool $(T \sim 20 \mathrm{~K})$ dust are brighter relative to the flux from the rest of the galaxy.

To study the dust temperature gradient in NGC 1291 in more detail, Figure 6 displays circles of radii $2^{\prime}, 3^{\prime}, 4^{\prime}, 5^{\prime}$, and $6^{\prime}$ overlaid on the SPIRE $250 \mu \mathrm{m}$ image, along with the estimated temperature of the dust within each annular aperture, using the same blackbody fitting technique as before. (These annuli match the dust mass annuli calculations found in Table 1. Unlike Table 1, the data for Figure 6 are not convolved to the $160 \mu \mathrm{m}$ resolution.) We remind the reader that such temperatures are representative, characterizing large grains that dominate the emission at wavelengths greater than $70 \mu \mathrm{m}$, and that, in fact, such large annuli contain a wide range of temperatures, including small grains undergoing temperature fluctuations. Keeping this in mind, if all disks, with or without rings, have similar dust temperature gradients due to the gradually increasing distance between star formation regions with radius, then we should be able to identify the same drop in temperature for spirals other than NGC 1291. We choose, from the KINGFISH sample, NGC 628 (SAc; $d=7.3 \mathrm{Mpc}$ ), which does not contain any rings but is face-on, is at a similar distance to NGC 1291, and has a similar angular size and total infrared luminosity to NGC 1291. We show in Figure 6 the dust temperatures for this galaxy for the same annular apertures as NGC 1291. For this galaxy, we see a gradual decline in temperature with some scatter. We see neither the dramatic dip in temperature at $3^{\prime}$ that is seen for NGC 1291 nor do we see such a large spread in temperatures, with NGC 628 having a temperature difference between inner and outer areas of $4.8 \mathrm{~K}$ versus $8.2 \mathrm{~K}$ for NGC 1291. Thus, it appears that the dust temperature from the ring is cooler than would be expected from a simple temperature decline with radius for a normal (non-ringed) spiral galaxy. Comparisons of spatially resolved dust temperatures with a larger number of KINGFISH galaxies are shown in M. Galametz et al. (2012, in preparation).

\subsection{AGN Heating}

The next possibility is that non-stellar sources such as an AGN could heat the dust in the inner portion of the galaxy, where we find a warmer dust temperature compared to the outer ring. This is suggested by the fact that outer rings are found to be much more frequent in galaxies with Seyfert nuclei (Hunt \& Malkan 1999). NGC 1291 is an X-ray source, with emission detected within 2'.5 (Bregman et al. 1995), which appears to be consistent with two energy components with their origin in a stellar component and an extended hot gas component (Hogg et al. 2001). Irwin et al. (2002) found an excess of soft emission similar to that seen in several other low-luminosity AGNs. Moustakas et al. (2010) classify NGC 1291 as an "AGN" type galaxy based on optical spectra of the nuclear region, although they note that one or more emission line(s) failed their $\mathrm{S} / \mathrm{N}>2$ requirement for such a classification. Recent analysis of X-ray spectra of the nuclear source also indicates a low-luminosity AGN with moderate obscuration (Luo et al. 2012), which could be a source of dust heating.

\subsection{Transport via the Bar}

The third possibility is that the bar in NGC 1291, seen at the various wavelengths in Figure 1, funnels gas into the inner 
region of the galaxy, replenishing the supply necessary for star formation. This compact region of star formation could then generate warmer dust temperatures than in the outer ring (see also Engelbracht et al. 2010). If gas is being transported inward in this manner, the observational evidence would likely be in the form of "hot spots" at the ends of the bar-features that are brighter than the rest of the bar formed by shock heating of the gas as it is drawn to the center of the galaxy, causing a "pileup" near the inner Lindblad resonance (Combes \& Gerin 1985; Shlosman et al. 1989). This is not seen in the H I data, which has a hole at the center (van Driel et al. 1988), or in the other wavelengths that are available. However, we cannot rule out this possibility, as such hot spots are thought to be small, very faint features. Hunt \& Malkan (1999) argue that rings alone could be signs of inward transport of material, essentially a second "pileup" of dust and gas in the outer resonance of the galaxy; the star formation in the ring prominent in the UV, presumably indicating stellar ages of $\sim 100 \mathrm{Myr}$, could be a residual signature of such a buildup. We note that transport of material via the bar may be related to the AGN hypothesis in the previous section, in that AGNs as well as star formation can be fueled via inflow.

\subsection{Stellar Age Gradient}

The last explanation for the change in cool dust temperature between the inner galaxy and outer ring is that the ring is composed mainly of an old population of stars with some current star formation. In this case, the dust in the ring was produced by generations of stars in the past and is heated now mainly by the old ( $\sim 10$ Gyr) stellar population, shown best by the $3.6 \mu \mathrm{m}$ image (Buta et al. 2010 and Figure 1) where the ring is a large, diffuse entity spanning much more physical space than seen at far-infrared wavelengths. Such "old population rings" have been suggested by comparisons between $B$ - and $I$-band images of nearby galaxies. Buta (1995) showed a typical galaxy of this type (IC 1438) which has a nuclear ring, an oval inner ring, and an outer ring that is much brighter in the $I$ passband than in the $B$. The explanation given for this difference in brightness was that the outer ring component formed first and left behind a stellar remnant as the other two inner features formed. An old outer ring would be unusual in that the dynamical timescales for forming rings or arms are much shorter at smaller disk radii (e.g., Freeman et al. 2010).

A remnant of this type could be the source of dust heating for the emission we see at far-infrared wavelengths. In addition, there would also be a contribution to the dust heating from ionizing ultraviolet photons, which have escaped from the existing (but weaker, in terms of contribution) star-forming regions. This combination of dust heating sources has been well modeled by, e.g., Misiriotis et al. (2001) and Popescu et al. (2002, 2011), and proposed to explain observations of other nearby galaxies (Hinz et al. 2004; Tabatabaei et al. 2007; Bendo et al. 2010). (Though see Groves et al. 2012 for a case where the old stellar population heats dust to warm temperatures.) Inspection of the $B-I$ image (R. J. Buta 2010, private communication) shows such a color difference and will be investigated further in R. J. Buta et al. (2012, in preparation). Simulations by Freeman et al. (2010) addressing Hoag's object, an outer ring galaxy with no inner morphological features, show that an outer ring can be formed via perturbations from a bar which then dissipates slowly over time. In the environment of these instabilities, the majority of the simulated gas particles fall to the center, while the remaining particles create an outer ring, with the region between the two essentially empty. If this is the manner in which NGC 1291 is forming its ring, then the implication is that the ring is unlikely to be older than the inner regions.

Noll et al. (2009) use SED-fitting techniques to study the star formation history of NGC 1291 and posit two exponential starburst events for the galaxy, one of which occurred $10 \mathrm{Gyr}$ ago and the second of which occurred $200 \mathrm{Myr}$ ago. This analysis was performed for the entire galaxy. A similar study for the inner portion and the ring separately would help disentangle their stellar histories.

\section{SUMMARY}

Far-infrared images from Herschel of the ringed galaxy NGC 1291 show that the morphology of the galaxy changes from that found in the optical, with some features such as the lens disappearing and other features such as the spiral-like inner region identified for the first time. Additionally, the large outer ring becomes more prominent at wavelengths longer than $160 \mu \mathrm{m}$. An exploration of temperature of the dust emission via blackbody fitting in annular apertures over the face of the galaxy yields the result that the dust in the ring is $\sim 6 \mathrm{~K}$ cooler than that found in the inner $\sim 3^{\prime}$. Further modeling shows that this cool dust in the ring dominates the total galaxy dust mass. We discuss whether, despite the presence of recent star formation traced by UV emission, the dust in the ring is heated primarily by a large, diffuse stellar population that is older than that of the inner portion of the galaxy and the bulge, lens, and bar features located there. This older stellar population would allow the cool dust to remain at temperatures of $\sim 20 \mathrm{~K}$. Other possibilities for the dust temperature difference discussed here include an expected temperature gradient due to the change in starlight heating intensity, heating of the inner region by a low-luminosity AGN, and increased star formation in the inner region due to gas transport along the bar.

J.L.H. thanks R. Buta for a preview of his $B$ and $I$ images of NGC 1291 and for helpful discussions. The following institutes have provided hardware and software elements to the SPIRE project: University of Lethbridge, Canada; NAOC, Beijing, China; CEA Saclay, CEA Grenoble, and OAMP in France; IFSI, Rome, and University of Padua, Italy; IAC, Tenerife, Spain; Stockholm Observatory, Sweden; Cardiff University, Imperial College London, UCL-MSSL, STFC-RAL, UK ATC Edinburgh, and the University of Sussex in the UK. Funding for SPIRE has been provided by the national agencies of the participating countries and by internal institute funding: CSA in Canada; NAOC in China; CNES, CNRS, and CEA in France; ASI in Italy; MCINN in Spain; Stockholm Observatory in Sweden; STFC in the UK; and NASA in the U.S.A. Additional funding support for some instrument activities has been provided by ESA. This research has made use of the NASA/IPAC Extragalactic Database (NED) which is operated by the Jet Propulsion Laboratory, California Institute of Technology, under contract with the National Aeronautics and Space Administration.

\section{REFERENCES}

Aniano, G., Draine, B. T., Calzetti, D., et al. 2012, ApJ, in press (arXiv:1207.4186)

Aniano, G., Draine, B. T., Gordon, K. D., \& Sandstrom, K. 2011, PASP, 123, 1218

Appleton, P. N., \& Struck-Marcell, C. 1996, Fundam. Cosm. Phys., 16, 111

Bagley, M., Minchev, I., \& Quillen, A. C. 2009, MNRAS, 395, 537 
Bendo, G. J. 2006, in ASP Conf. Ser. 357, The Spitzer Space Telescope: New Views of the Cosmos, ed. L. Armus \& W.T. Reach (San Francisco, CA: ASP), 192

Bendo, G. J., Wilson, C. D., Pohlen, M., et al. 2010, A\&A, 518, L65

Bregman, J. N., Hogg, D. E., \& Roberts, M. S. 1995, ApJ, 441, 561

Brook, C., Richard, S., Kawata, D., Martel, H., \& Gibson, B. K. 2007, ApJ, 658, 60

Buta, R. J. 1995, ApJS, 96, 39

Buta, R. J. 2011, in Planets, Stars, and Stellar Systems, Vol. 6, ed. W. C. Keel (Springer Reference), in press (arXiv:1102.0550)

Buta, R. J., \& Combes, F. 1996, Fundam. Cosm. Phys., 17, 95

Buta, R. J., Sheth, K., Regan, M., et al. 2010, ApJS, 190, 147

Caldwell, N., Kennicutt, R., Phillips, A. C., \& Schommer, R. A. 1991, ApJ, 370, 526

Calzetti, D., Wu, S.-Y., Hong, S., et al. 2010, ApJ, 714, 1256

Ciardullo, R., Rubin, V. C., Jacoby, G. H., Ford, H. C., \& Ford, W. K. 1988, AJ, 95, 438

Combes, F., \& Gerin, M. 1985, A\&A, 150, 327

Crocker, D. A., Baugus, P. D., \& Buta, R. 1996, ApJS, 105, 353

Dale, D. A., Aniano, G., Engelbracht, C. W., et al. 2012, ApJ, 745, 95

Dale, D. A., Gil de Paz, A., Gordon, K. D., et al. 2007, ApJ, 655, 863

de Jong, R. S., Seth, A. C., Radburn-Smith, D. J., et al. 2007, ApJ, 667, L49

de Vaucouleurs, G. 1975, ApJS, 29, 193

de Vaucouleurs, G., de Vaucouleurs, A., Corwin, H. G., et al. 1991, Third Reference Catalogue of Bright Galaxies (New York: Springer)

Devereux, N., Jacoby, G. H., \& Ciardullo, R. 1995, AJ, 110, 1115

Donovan, J. L., Serra, P., van Gorkom, J. H., et al. 2009, AJ, 137, 5037

Draine, B. T., Dale, D. A., Bendo, G., et al. 2007, ApJ, 663, 866

Draine, B. T., \& Li, A. 2001, ApJ, 551, 807

Draine, B. T., \& Li, A. 2007, ApJ, 657, 810

Engelbracht, C. W., Blaylock, M., Su, K. Y. L., et al. 2007, PASP, 119, 994

Engelbracht, C. W., Gordon, K. D., Bendo, G. J., et al. 2004, ApJS, 154, 248

Engelbracht, C. W., Hunt, L. K., Skibba, R. A., et al. 2010, A\&A, 518, L56

Fazio, G. G., Hora, J. L., Allen, L. E., et al. 2004, ApJS, 154, 10

Freeman, T., Howard, S., \& Byrd, G. G. 2010, Celest. Mech. Dyn. Astron., 108, 23

Galametz, M., Kennicut, R. C., Albrecht, M., et al. 2012, MNRAS, in press (arXiv:1207.1301)

Gil de Paz, A., Boissier, S., Madore, B. F., et al. 2007, ApJS, 173, 185

Gil de Paz, A., Madore, B. F., Boissier, S., et al. 2005, ApJ, 627, L29

Gordon, K. D., Engelbracht, C. W., Fadda, D., et al. 2007, PASP, 119, 1019

Gordon, K. D., Rieke, G. H., Engelbracht, C. W., et al. 2005, PASP, 117, 503

Griffin, M. J., Abergel, A., Abreu, A., et al. 2010, A\&A, 518, L3

Groves, B., Krause, O., Sandstrom, K., et al. 2012, MNRAS, Accepted (arXiv:1206.2925)

Hinz, J., Gordon, K., \& Rieke, G. 2008a, Spitzer Proposal ID 489

Hinz, J. L., Misselt, K., Rieke, M. J., et al. 2006, ApJ, 651, 874

Hinz, J. L., Rieke, G. H., Gordon, K. D., et al. 2004, ApJS, 154, 259

Hinz, J. L., Rieke, M. J., Rieke, G. H., et al. 2008b, ApJ, 663, 895
Hogg, D. E., Roberts, M. S., Bregman, J. N., \& Haynes, M. P. 2001, AJ, 121, 1336

Hunt, L. K., \& Malkan, M. A. 1999, ApJ, 516, 660

Irwin, J. A., Sarazin, C. L., \& Bregman, J. N. 2002, ApJ, 570, 152

Kannappan, S. J., Guie, J. M., \& Baker, A. J. 2009, AJ, 138, 579

Kennicutt, R. C., Calzetti, D., Aniano, G., et al. 2011, PASP, 123, 1347

Kennicutt, R. C., Jr. 1989, ApJ, 344, 685

Kennicutt, R. C., Jr. 1998, ApJ, 498, 541

Kennicutt, R. C., Jr., Armus, L., Bendo, G., et al. 2003, PASP, 115, 928

Kennicutt, R. C., Jr., Lee, J. C., Funes, S. J., et al. 2008, ApJS, 178, 247

Li, J.-T., Wang, Q. D., Li, Z., \& Chen, Y. 2011, ApJ, 737, 41

Luo, B., Fabbiano, G., Fragos, T., et al. 2012, ApJ, 749, 130

Marino, A., Bianchi, L., Rampazzo, R., et al. 2011, ApJ, 736, 154

Masters, K. L. 2005, PhD thesis, Cornell Univ.

McConnachie, A. W., Irwin, M. J., Ibata, R. A., et al. 2009, Nature, 461, 66

Meurer, G., Hanish, D. J., Ferguson, H. C., et al. 2006, ApJS, 165, 307

Michard, R., \& Marchal, J. 1993, A\&AS, 98, 29

Misiriotis, A., Popescu, C. C., Tuffs, R., \& Kylafis, N. D. 2001, A\&A, 372, 775

Moustakas, J., Kennicutt, R. C., Jr., Tremonti, C. A., et al. 2010, ApJS, 190, 233

Muñoz-Mateos, J. C., Gil de Paz, A., Boissier, S., et al. 2007, ApJ, 658, 1006

Noll, S., Burgarella, D., Giovannoli, E., et al. 2009, A\&A, 507, 1793

Ott, S. 2010, in ASP Conf. Ser. 434, in Astronomical Data Analysis Software and Systems XIX, ed. Y. Mizumoto, K.-I. Morita, \& M. Ohishi (San Francisco, CA: ASP), 139

Perrine, C. D. 1922, MNRAS, 82, 486

Pérez, I., \& Freeman, K. 2006, A\&A, 454, 165

Planck Collaboration, Ade, P. A. R., Aghanim, N., et al. 2011, A\&A, 536, A17

Poglitsch, A., Waelkens, C., Geis, N., et al. 2010, A\&A, 518, L2

Pohlen, M., Cortese, L., Smith, M. W. L., et al. 2010, A\&A, 518, L72

Popescu, C. C., Tuffs, R. J., Dopita, M. A., et al. 2011, A\&A, 527, A109

Popescu, C. C., Tuffs, R. J., Völk, H. J., Pierini, D., \& Madore, B. F. 2002, ApJ, 567,221

Prescott, M. K. M., Kennicutt, R. C. Jr, Bendo, G. J., et al. 2007, ApJ, 668, 182 Rautiainen, P., \& Salo, H. 2000, A\&A, 362, 465

Rieke, G. H., Young, E. T., Engelbracht, C. W., et al. 2004, ApJS, 154, 25

Roussel, H. 2012, arXiv:1205.2576

Roussel, H., Wilson, C. D., Vigroux, L., et al. 2010, A\&A, 518, L66

Sandstrom, K., Krause, O., Linz, H., et al. 2010, A\&A, 518, L59

Schlegel, D. J., Finkbeiner, D. P., \& Davis, M. 1998, ApJ, 500, 525

Schwarz, M. P. 1981, ApJ, 247, 77

Sheth, K., Regan, M., Hinz, J. L., et al. 2010, PASP, 122, 1397

Skibba, R. A., Engelbracht, C. W., Dale, D., et al. 2011, ApJ, 738, 89

Shlosman, I., Frank, J., \& Begelman, M. C. 1989, Nature, 338, 45

Stansberry, J. A., Gordon, K. D., Bhattacharya, B., et al. 2007, PASP, 119, 1038

Tabatabaei, F. S., Beck, R., Krause, M., et al. 2007, A\&A, 466, 509

Thilker, D. A., Bianchi, L., Meurer, G., et al. 2007, ApJS, 173, 538

Thilker, D. A., Bianchi, L., Schiminovich, D., et al. 2010, ApJ, 714, L171

Thilker, D. A., Hoopes, C. G., Bianchi, L., et al. 2005, ApJ, 619, L67

van Driel, W., Rots, A. H., \& van Woerden, H. 1988, A\&A, 204, 39

Wiebe, D. V., Ade, P. A. R., Bock, J. J., et al. 2009, ApJ, 707, 1809 